\title{
The True-Positive Rate of a Screening Questionnaire for Temporoman- dibular Disorders
}

\author{
Akira Nishiyama*, Natsuko Otomo, Kaori Tsukagoshi, Shoko Tobe and Koji Kino
}

Section of Temporomandibular Joint and Oral Function, Department of Comprehensive Patient Care, Graduate School, Tokyo Medical and Dental University, Tokyo, Japan

\begin{abstract}
Background: Temporomandibular disorders (TMD) occur at an incidence of 5-12\% in the general population. We aimed to investigate the rate of true-positives for a screening questionnaire for TMD (SQ-TMD) and differences in the characteristics between the true-positive and false-negative groups.

Materials and Methods: Seventy-six individuals (16 men, 60 women; mean age, $41.1 \pm 16.5$ years) were selected from patients with TMD who had visited the Temporomandibular Joint Clinic at Tokyo Medical and Dental University. The patients were assessed using a questionnaire that contained items on TMD screening (SQ-TMD); pain intensity (at rest, maximum mouth-opening, and chewing), as assessed using the visual analog scale (VAS); and TMD-related limitations of daily function (LDF-TMD). A logistic regression analysis was performed to assess the factors potentially influencing the true-positive rate.

Results: Of the 76 subjects, $62(81.6 \%)$ were true-positive for the questionnaire based on the SQ-TMD scores. The mean VAS score for maximum mouth-opening and chewing and the mean LDF-TMD score were significantly greater in the true-positive group than those in the false-negative group. The results of the logistic regression analysis showed that only the VAS score for chewing was a statistically significant factor $(P<0.05)$.

Conclusion: The true-positive rate of TMD using SQ-TMD was very high. The results indicate that SQ-TMD can be used to screen TMD in patients with moderate or severe pain and difficulty in living a healthy daily life.
\end{abstract}

Keywords: Limitations of daily function, pain intensity, sensitivity, true-positive rate, visual analog scale.

\section{INTRODUCTION}

Temporomandibular disorders (TMD) are a subclass of musculoskeletal disorders resulting from the dysfunction of the stomatognathic system and affect the masticatory muscles, temporomandibular joint, and orofacial structures [1]. TMD have been identified as the main cause of nondental pain in the orofacial region, and the most common symptoms are jaw and facial pain [1].

TMD are a significant public health problem affecting approximately $5-12 \%$ of the population [2]. In Japan, the prevalence of TMD among patients making a first visit to the dental office has been reported to be $11.7 \%$ [3].

The Research Diagnostic Criteria for TMD (RDC/TMD) has been adopted worldwide for the diagnosis of TMD [4]. However, patients with TMD might not receive appropriate treatment because of a general lack of awareness of TMD at an institution or the failure to visit a hospital knowledgeable on TMD or the RDC/TMD criteria. Furthermore, the RDC/TMD comprise a number of questions, and the

*Address correspondence to this author at the 1-5-45, Yushima, Bunkyo-ku, Tokyo 113-8549, Japan; Tel: +81-3-5803-5713; Fax: +81-3-5803-5713;

E-mail: anishi.tmj@tmd.ac.jp exploratory items are not adapted for use in large epidemiologic studies and dental checkups in school and the workplace. For addressing these issues, the development of tools that can be used to screen TMD easily within any institution is needed.

Several questionnaires for use in epidemiologic studies of TMD have been reported; however, the validation of the questionnaires in these studies was limited [5-8]. In addition, no reports have been published on the use of these questionnaires for TMD screening. Only the previous study of Sugisaki et al. [3] has reported a screening questionnaire for TMD (SQ-TMD). This screening questionnaire was developed to screen for TMD in adults and comprises 4 items that are scored based on a 5-point numeric rating scale. The study aim was to investigate the rate of true-positives for the SQTMD and the differences in the characteristics between the true-positive and false-negative groups to clarify the patient characteristics for efficient screening by the SQ-TMD.

\section{MATERIALS AND METHODS}

\section{Subjects}

Seventy-six individuals (16 men, 60 women; mean age, $41.1 \pm 16.5$ years) were selected from patients with TMD 
Table 1. Comparison of patient characteristics between the true-positive and false-negative groups.

\begin{tabular}{|c|c|c|c|}
\hline & True-Positive & False-Negative & \multicolumn{1}{c|}{} \\
\hline \hline $\mathrm{N}(\%)$ & $62(81.6)$ & $14(18.4)$ & 0.979 \\
\hline Age, year (SD) & $41.1(16.3)$ & $41.0(18.3)$ & 0.445 \\
\hline Women (\%) & $50(80.6)$ & $10(71.4)$ & \\
\hline
\end{tabular}

$\mathrm{SD}$, standard deviation.

who had visited the Temporomandibular Joint Clinic at Tokyo Medical and Dental University for treatment between August and December 2012. The inclusion criteria were age $>19$ years and a diagnosis of TMD based on the RDC/TMD. The exclusion criteria were 1) pain associated with acute inflammation of the stomatognathic system; 2) chronic arthritis such as rheumatoid arthritis; 3) missing teeth and/or the use of a removable denture, but having a fixed partial denture restoration over 1 year; and 4) the use of regular medication such as analgesics, anti-anxiety drugs, antidepressants, and psychotropics. The Institutional Review Board approved the study protocol (No. 787), which complied with the Helsinki Declaration of 1975, as revised in 1983. Informed consent was obtained from all enrolled participants.

\section{Questionnaire}

The questionnaire used in this study included the following: age, sex, the SQ-TMD (4 items), the TMD-related limitation of daily functions (10 items), and pain intensity (at rest, maximum mouth-opening, and chewing).

The SQ-TMD, developed by Sugisaki et al. [3], was used to screen patients for TMD. The following 4 items are included in the SQ-TMD: 1) If you open your mouth wide, can you fit 3 fingers held vertically in your mouth? 2) Do you experience pain in the face, jaw, temple, or in front of the ear when you open and close your mouth? 3) Can you open your mouth without any deviation? 4) Do you experience pain in the face, jaw, temple, or in front of the ear when you eat chewy foods such as beef jerky, dried cuttlefish, or octopus? For each item, the subject chose 1 of 5 levels on a numerical rating scale from "no problem at all" ( 0 points) to "extremely difficult" (4 points). In addition, a different questionnaire by Sugisaki et al. [9] was used for the TMD-related limitation of daily functions (LDF-TMD). This component of the questionnaire consisted of the question "How much does your present jaw problem prevent or limit you from the following daily activities?: 1) opening your mouth when you eat big pieces of food; 2) grinding thin food; 3) clenching your teeth; 4) brushing your back teeth; 5) yawning; 6) talking for a long period; 7) using your jaw for a long period during meals; 8) performing activities at home, school, and/or work; 9) falling asleep soon after going to bed; and 10) sleeping continuously at night." The subjects rated these 4 items by using the same 5-point numeric rating scale as above. The summary score of the 10 items, ranging from 0 to 40 points, was used for analysis.
Pain intensity was estimated by using a visual analog scale (VAS) consisting of a 100-mm line with "no pain" on the extreme left and "intolerable pain" on the extreme right. The subjects rated the severest TMD-related pain experienced at rest, maximum mouth-opening, and chewing.

\section{Statistical Analysis}

The rate of true-positives and false-negatives were calculated from the results of the SQ-TMD. A cutoff value of 4.5 for the total score from items 1-4 was used to assign patients to the true-positive and false-negative groups: participants with a score of $\geq 5.0$ or $\leq 4.0$ were assigned to the truepositive or false-negative group, respectively.

The paired Student $t$-test and $\chi^{2}$ test were used to analyze the differences between the true-positive and false-negative data. The $\chi^{2}$ test was used to analyze the differences in the true-positive group based on RDC/TMD categorization of the patients into groups 1 (muscle disorders), 2 (disk displacements), and 3 (arthralgia, arthritis, and arthrosis). The factors potentially influencing the true-positive rate were assessed by logistic regression analyses using odds ratios and 95\% confidence intervals as measures of association. The dependent variable was the result of the TMD screening (false-positive, 0; true-positive, 1). The independent variables were age, sex, the various VAS scores, and the LDFTMD score. The covariates were entered into the logistic regression analysis by a stepwise forward technique, with $P<0.05$ considered statistically significant. All statistical analyses were performed using the Statistical Package for Social Sciences (SPSS version 21.0; Tokyo, Japan).

\section{RESULTS}

Of the 76 subjects, $62(81.6 \%)$ were true-positive for the SQ-TMD based on the total scores from items 1-4. No significant differences in age and sex were found between the true-positive and false-negative groups (Table $\mathbf{1}$ ).

The differences were not statistically significant between the RDC/TMD groups (Table 2 ).

The mean VAS score at maximum mouth-opening and chewing and the mean LDF-TMD score were significantly greater in the true-positive group relative to those in the false-negative group (Table 3). According to the results of the logistic regression analysis, only the VAS score for chewing was a statistically significant factor $(P<0.05$; Table 4). 
Table 2. Rate and number of true-positives in tmd screening questionnaire.

\begin{tabular}{|c|c|c|c|}
\hline \multirow{2}{*}{ RDC/TMD } & \multicolumn{2}{|c|}{ True-Positive } & \multirow{2}{*}{$P$} \\
\hline & Number & $\%$ & \\
\hline Group $1(\mathrm{~N}=5)$ & 4 & 80 & \multirow{3}{*}{0.422} \\
\hline Group $2(\mathrm{~N}=56)$ & 44 & 78.6 & \\
\hline Group $3(\mathrm{~N}=15)$ & 14 & 93.3 & \\
\hline Total & 62 & 81.6 & \\
\hline
\end{tabular}

TMD, temporomandibular disorders; RDC/TMD, research diagnostic criteria for TMD.

$*, \chi^{2}$ test.

Table 3. Comparison of true-positive and false-negative groups in the tmd screening questionnaire.

\begin{tabular}{|c|c|c|c|}
\hline \multirow{2}{*}{} & \multirow{2}{*}{ Total } & \multicolumn{2}{|c|}{ TMD Screening Questionnaire } \\
\cline { 2 - 4 } & & $\begin{array}{c}\text { True-Positive } \\
\text { N=62 }\end{array}$ & $\begin{array}{c}\text { False-Negative } \\
\text { N=14 }\end{array}$ \\
\hline \hline Mean pain VAS, mm (SD) & & & $7.3(12.4)$ \\
\hline At rest & $10.6(16.3)$ & $11.3(17.1)$ & $26.1(28.7)$ \\
\hline Maximum mouth-opening & $53.0(26.8)$ & $59.1(22.4)$ & $4.4(7.3)$ \\
\hline Chewing & $40.0(31.4)$ & $48.1(29.1)$ & $<0.001$ \\
\hline LDF-TMD, score (SD) & $11.5(5.5)$ & $12.7(5.1)$ & $5.8(3.6)$ \\
\hline
\end{tabular}

TMD, temporomandibular disorders; SD, standard deviation; VAS, visual analog scale; LDF-TMD, limitations of daily functions in TMD.

Table 4. Results of logistic regression analysis.

\begin{tabular}{|c|c|c|c|}
\hline & $\boldsymbol{P}$ & Odds Ratio & 95\% Confidence Interval \\
\hline \hline Pain intensity at chewing & 0.003 & & $1.08-1.46$ \\
\hline 1 -mm increments & & 1.25 & $2.66-3.60$ \\
\hline 5-mm increments & & 3.10 & $8.24-11.1$ \\
\hline $10-\mathrm{mm}$ increments & & 9.58 & 2 \\
\hline
\end{tabular}

Note: Only the significant factor is shown.

\section{DISCUSSION}

In the present study, no significant differences were found between the true-positive and false-negative groups in terms of age and sex. Women have been found to predominate among patients requiring treatment for TMD [10], and a relationship between female sex and painful TMD symptoms has been widely reported in the literature [11-13]. Based on the high true-positive rate observed in the present study, our results indicate that the SQ-TMD can be used to screen for TMD without age or sex influencing the process. Similarly, the absence of significant differences in the true-positive rate between the RDC/TMD groups suggests that the TMD pathology will not influence the screening process.

In developing the SQ-TMD, Sugisaki et al. [3] extracted items 1 - 4 from a 20-item questionnaire administered to dental patients. Based on a cutoff value of 4.5 for the total score from these items, the participants with a score of $\geq 5.0$ or $\leq 4.0$ were assigned to the TMD or non-TMD group, respectively. The sensitivity, specificity, and false-positive rate derived from the TMD screening were $0.746,0.811$, and 0.189 , respectively. Dworkin and LeResche [4] had previously reported that a sensitivity of $\geq 0.7$ was required to diag- 
nose TMD at a prevalence of $10 \%$. The results of Sugisaki et al. [3] were consistent with the findings of this previous study. Moreover, in the present study, the true-positive rate of TMD was $81.6 \%$ (sensitivity, 0.816), resulting in a sensitivity similar to that observed in the study of Sugisaki et al. [3].

According to Collins et al. [14], a patient with a baseline VAS score exceeding 30 or $54 \mathrm{~mm}$ will likely be experiencing at least moderate or severe pain, respectively. In the truepositive group, the mean VAS scores for maximum mouthopening and chewing were 59.1 and $48.1 \mathrm{~mm}$, respectively, and were shown to represent severe and moderate pain, respectively. The VAS scores for the false-negative group were each $<30 \mathrm{~mm}$ and shown to represent very light pain. Moreover, the mean LDF-TMD score in the true-positive group was 2-fold or more compared with that of the falsenegative group. Based on these results, it is possible that the SQ-TMD can be used to detect TMDs in patients with moderate or severe pain or with difficulty in living a healthy daily life.

Moufti et al. [15] ranked items for TMDs in the Oral Health Impact Profile and reported that items related to chewing difficulty (i.e., sore jaw, difficulty chewing foods, discomfort while eating foods, and avoiding some foods) and limitations in daily functions (e.g., difficulty relaxing, feeling tense, and being upset) had a higher rank than other items. These results suggested that disabilities in chewing and daily functions are serious problems for patients with TMD. Consistent with this previous report, the logistic regression analysis in the present study found that chewing was a factor that affected the SQ-TMD screening results. In the true-positive group, the proportion of a score of 3 or 4 in the SQ-TMD in were $51.7 \%$ (item 1), $72.5 \%$ (item 2), 50.0\% (item 3), and $80.7 \%$ (item 4). Because item 4 was a question about chewing pain, it was considered that the VAS score of chewing pain had the greatest impact on the true-positive rate of TMD screening. The frequency with which the subjects reported that they were aware of mouth-opening pain was low, perhaps because they did not open their mouths widely enough for the questionnaire. However, it was possible that the subjects were likely to be aware of the pain in their daily lives, because chewing pain was experienced at every meal.

SQ-TMD can be used to determine the potential presence of TMD with a high true-positive rate. In particular, our findings indicate it is suitable for screening TMD in patients with severe pain during jaw function (mouth-opening or chewing) or with a hindrance in daily life. However, because the SQTMD was not developed to screen for TMD in adolescents, but in adults, the sensitivity and specificity for this age group is unknown. Hence, in the future, a new questionnaire should be developed to screen for TMD in adolescents.

Our results suggest that the SQ-TMD can be completed in a short time because it is composed of only a few question and can be used in group checkups within a region, school, or workplace to detect patients with TMD symptoms. Additionally, this questionnaire can permit an initial diagnosis in a general hospital with poor knowledge of TMD and thereby provide the patient with an opportunity to visit a professional medical organization for patients with TMD symptoms. Finally, the use of this screening questionnaire in large epidemiologic studies could enable the collection of large quantities of data and thus provide a significant contribution to TMD research.

\section{CONCLUSION}

Our study aimed to investigate the rate of true-positives for a SQ-TMD and the differences in the characteristics between the true-positive and false-negative groups.

The true-positive rate of TMD using the SQ-TMD was very high. The results indicate that SQ-TMD can be used to screen TMD in patients with moderate or severe pain and difficulty in living a healthy daily life.

\section{CONFLICT OF INTEREST}

The authors confirm that this article content has no conflict of interest.

\section{ACKNOWLEDGEMENTS}

We thank the subjects who participated in this study.

\section{REFERENCES}

[1] Okeson JP. Bell's orofacial pains: the clinical management of orofacial pain. $6^{\text {th }}$ ed. Quintessence Publishing: Chicago, IL 2005.

[2] National Institute of Dental and Craniofacial Research. Facial pain. Available fom: (http://www.nidcr.nih.gov/DataStatistics/FindDataBy-Topic/FacialPain/).

[3] Sugisaki M, Kuruma R, Kino K, et al. Selection of question items for screening patients with temporomandibular disorders and estimation of their validity. J Jpn Soc TMJ 2007; 19: 177-84.

[4] Dworkin SF, LeResche L. Research diagnostic criteria for temporomandibular disorders: review, criteria, examinations and specifications, critique. J Craniomandib Disord 1992; 6: 301-55.

[5] Helkimo M. Studies on function and dysfunction of the masticatory system. I. An epidemiological investigation of symptoms of dysfunction in Lapps in the north of Finland. Proc Finn Dent Soc 1974; 70: 37-49.

[6] Helkimo M. Studies on function and dysfunction of the masticatory system. II. Index for anamnestic and clinical dysfunction and occlusal state. Sven Tandlak Tidskr 1974; 67: 101-21.

[7] Helkimo M. Studies on function and dysfunction of the masticatory system. 3. Analysis of anamnestic and clinical recordings of dysfunction with the aid of indices. Sven Tandlak Tidskr 1974; 67: 165-81.

[8] Helkimo M. Studies on function and dysfunction of the masticatory system. IV. Age and sex distribution of symptoms of dysfunction of the masticatory system in Lapps in the north of Finland. Acta Odont Scand 1974; 32: 255-67.

[9] Sugisaki M, Kino K, Yoshida N, Ishikawa T, Amagasa T, Haketa T. Development of a new questionnaire to assess pain-related limitations of daily functions in Japanese patients with temporomandibular disorders. Comm Dent Oral Epidemiol 2005; 33: 384-95.

[10] McNeill C. The optimum temporomandibular joint condyle position in clinical practice. Int J Periodont Restorat Dent 1985; 5: 52 76.

[11] Cimino R, Farella M, Michelotti A, Pugliese R, Martina R. Does the ovarian cycle influence the pressure-pain threshold of the masticatory muscles in symptom-free women? J Orofac Pain 2000; 14 : 105-11. 
[12] Dao TT, LeResche L. Gender differences in pain. J Orofac Pain 2000; 14: 169-84.

[13] LeResche L, Mancl LA, Drangsholt MT, Huang G, Von Korff M. Predictors of onset of facial pain and temporomandibular disorders in early adolescence. Pain 2007; 129: 269-78.
[14] Collins SL, Moore RA, McQuay HJ. The visual analogue pain intensity scale: what is moderate pain in millimetres? Pain 1997; 72: 95-7.

[15] Moufti MA, Wassell RW, Meechan JG, Allen PF, John MT, Steele JG. The oral health impact profile: ranking of items for temporomandibular disorders. Eur J Oral Sci 2011; 119: 169-74.

Received: September 26, 2014

Revised: October 28, 2014

Accepted: November 11, 2014

(c) Nishiyama et al.; Licensee Bentham Open.

This is an open access article licensed under the terms of the Creative Commons Attribution Non-Commercial License (http://creativecommons.org/licenses/by-nc/3.0/) which permits unrestricted, non-commercial use, distribution and reproduction in any medium, provided the work is properly cited. 